\title{
construcción de espaldones
}

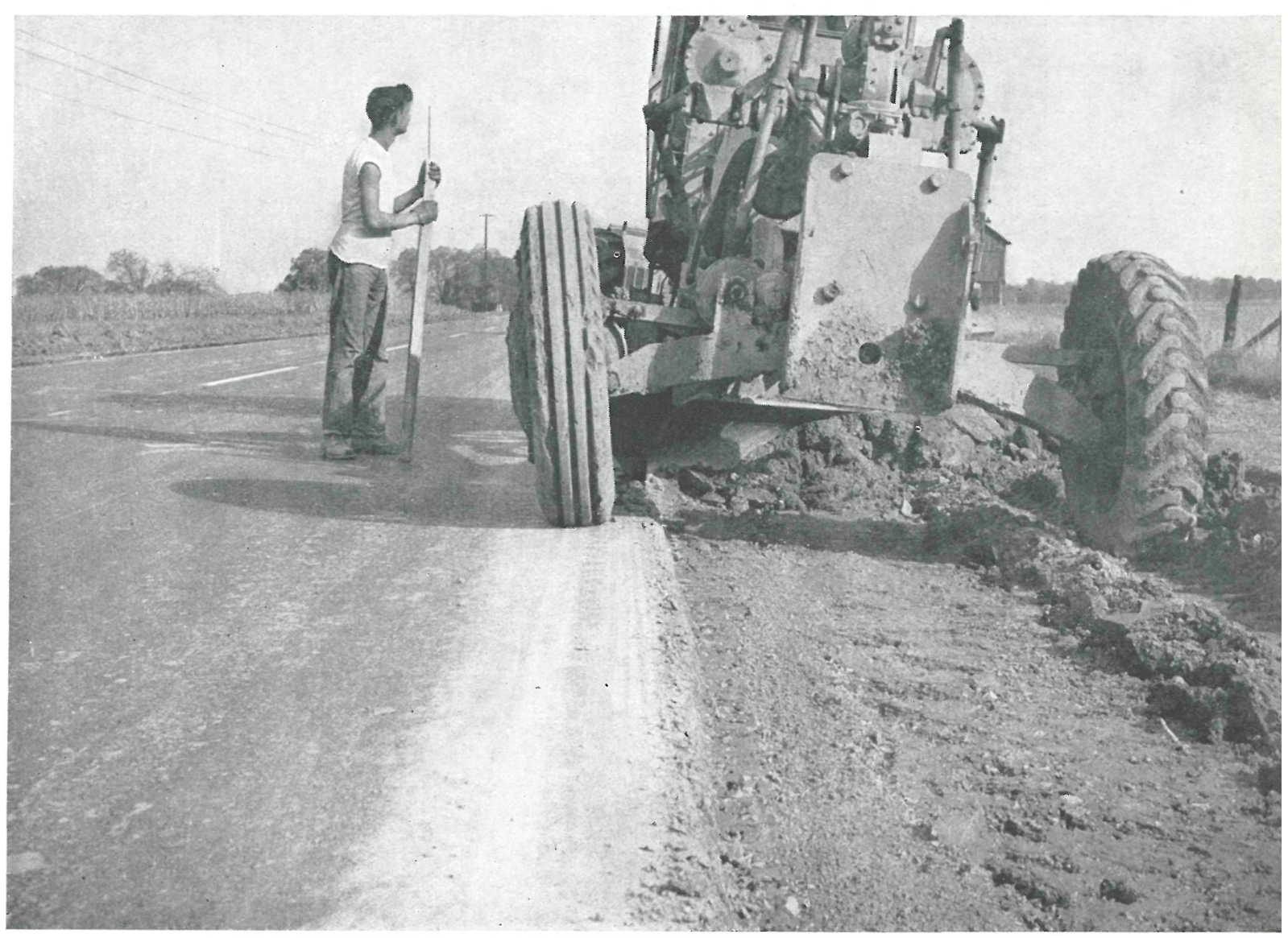

C. H. SHEPARD, ingeniero

$518 \cdot 1$

S I N O P S I S

Breve reseña del estudio sobre la construcción de espaldones en las carreteras, que el autor preparó para presentarlo después en el Symposium de la Asamblea anual de la Highway Research Board. El autor da cuenta de los procedimientos generales a este respecto empleados en la Jefatura de Carreteras de Ohio, de la que es ingeniero especializado en mecánica del suelo.

\section{Ideas generales sobre el apoyo del firme}

Hace ya mucho tiempo que se viene aceptando que, para la preparación de la subrasante y capas de cimiento de un firme, debe preverse un eficiente drenaje de la zona afectada. Recientemente se han mejorado mucho las prácticas hoy corrientes del drenado de las aguas de superficie. En las excavaciones han de tenerse en cuenta los drenes necesarios para evitar toda perturbación posible del terreno y cimiento durante la construcción, y con tanto mayor cuidado si se trata de una excavación en trinchera, por poca que sea la profundidad que ésta presente. 


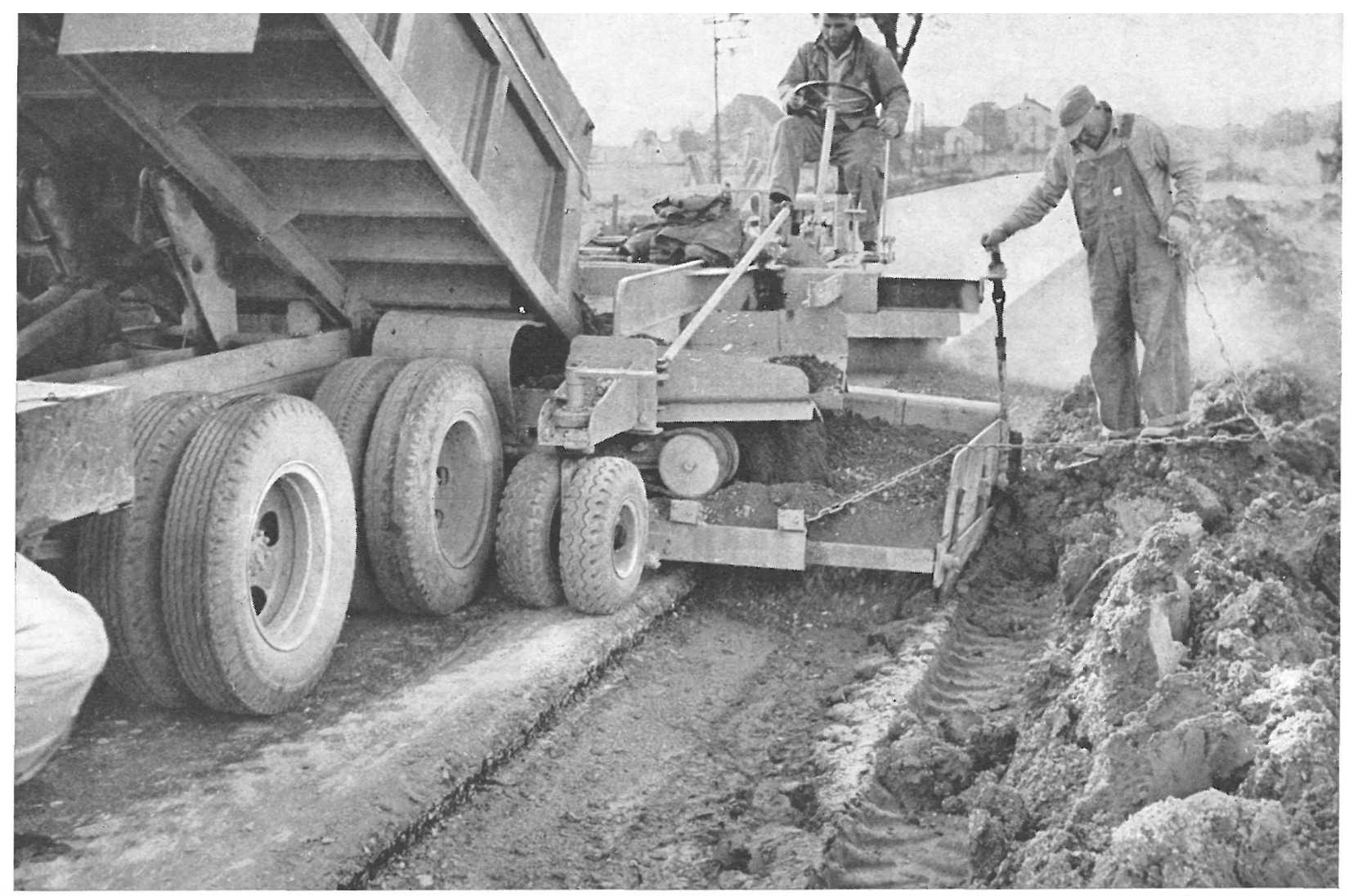

Relleno de la caja de un espaldón.

En los ensanches de caminos se hace sentir una tendencia muy generalizada, que consiste en preparar la plataforma y cimiento

a la vez que se abre la zanja para la caja de la banda del ensanche,

cuidando de dejar terminado el trozo en el día emprendido al finalizar la jornada.

En una excavación, plataforma o caja donde no se haya previsto un drenaje activo provisional para recoger y encauzar las aguas en cunetas o colectores subterráneos,

las consecuencias suelen ser devastadoras en casos de lluvias persistentes y fuertes, pues el terreno y cimiento se reblandecen y erosionan tanto

que hay que volver a readaptarlas a su estado pre:io, y, por consiguiente,

se pierde un lastimoso tiempo y esfuerzo.

Para amoldar la ejecución con las exigencias del dimensionado y regularidad del proyecto es necesario espesores uniformes.

En estos últimos años se han venido experimentando una serie de mejoras notables en el equipo empleado en la ejecución, logrando una uniformidad y exactitud en los espesores que cada vez se ajustan mejor a las exigencias previamente fijadas;

pero aún así no es suficiente, ya que los contratistas tienden con frecuencia a prestar menos cuidado del necesario para lograr un resultado que se halle dentro de las tolerancias y, por tanto,

se hace sentir la necesidad de una mecanización más depurada para dar

la forma exacta a la subrasante y capas de cimientos.

Si se quiere obtener una base de apoyo sana y estable, es necesario una consolidación muy uniforme del terreno y capas superiores del revestimiento;

por ello, durante la construcción se necesitan medios de control para conocer rápidamente las soluciones de continuidad que pudiera presentar la compactación, de la cual depende la estabilidad de la estructura del firme.

El medio de control más extendido, en este sentido, lo constituye el cilindro compactador de ruedas neumáticas, que se utiliza, no ya para la subrasante, sino para el cimiento y base inclusive. 


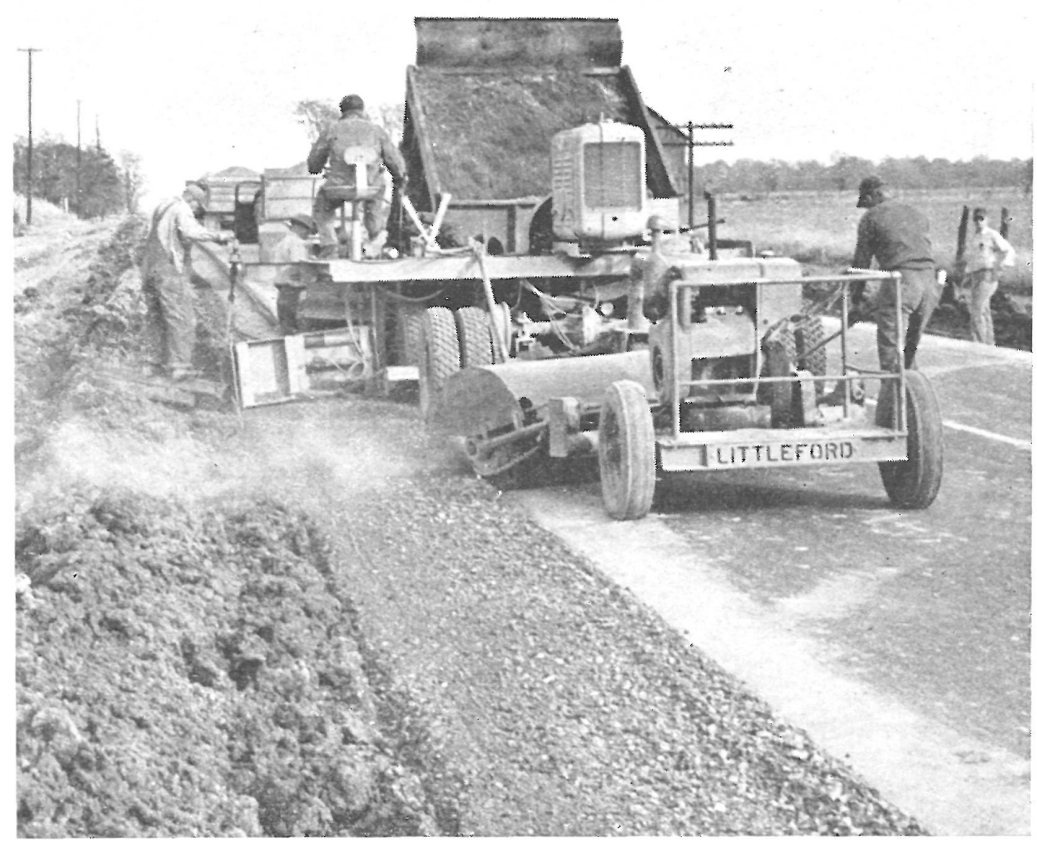

Rellenando la caja del espaldón con áridos machacados.

Compactación cilindro de ruedas neumáticas.

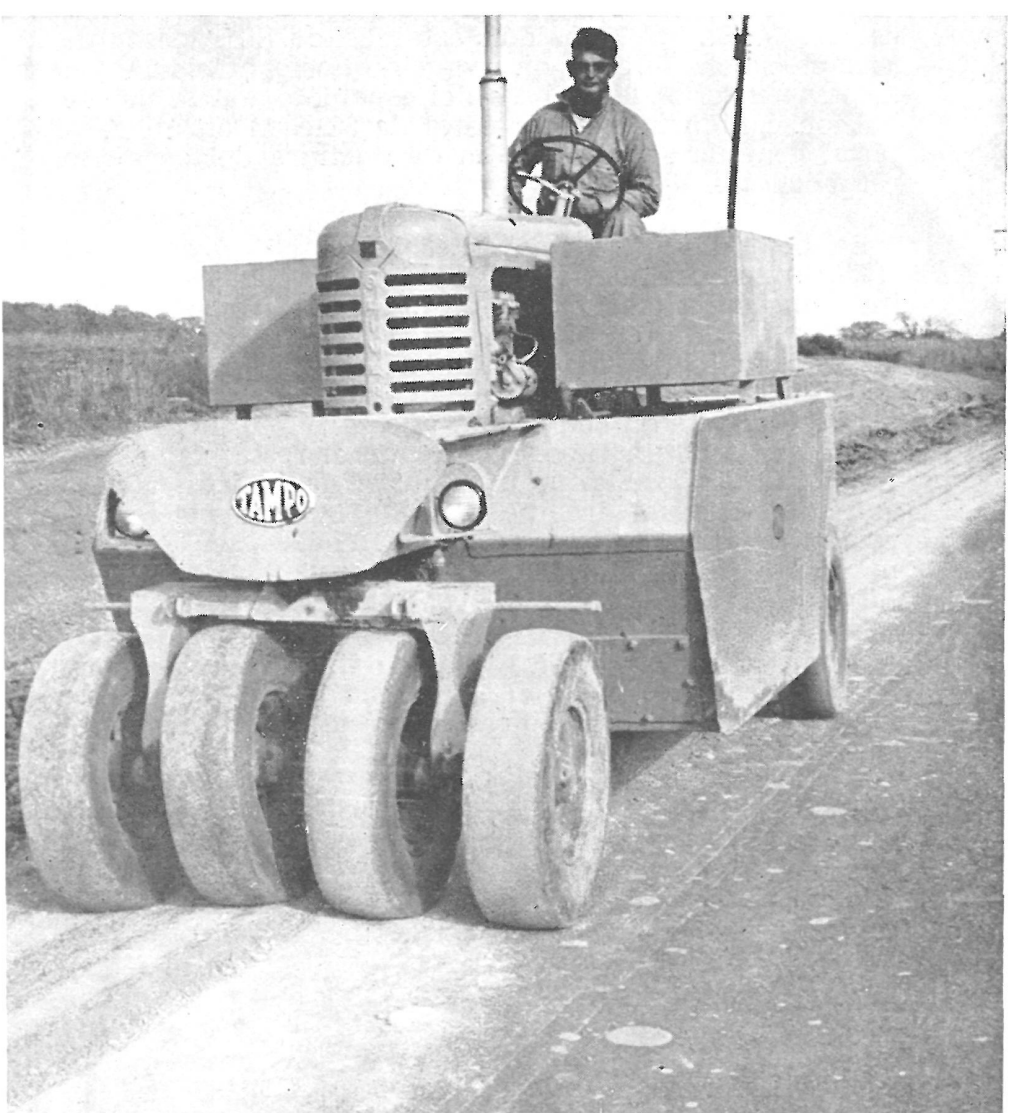

En Ohio se viene empleando el cilindro de cuatro ruedas neumáticas para la compactación antes indicada e, incluso, para las bases de macadam. La forma de operar ha sido reglamentada en la Jefatura de este Departamento. En estas instrucciones se especifica que el referido cilindro de ruedas neumáticas ha de poder variar su carga de 25 a 50 toneladas, y la presión de los neumáticos desde 6,32 hasta $10,54 \mathrm{~kg} / \mathrm{cm}^{2}$. En cada ensayo de estabilidad o regularidad de compactación son imprescindibles dos o más pruebas.

La utilización de estos ensayos permite conocer el estado de toda la superficie, comparando, después, las partes que han demostrado comportarse mejor con otra clase de ensayos generalmente empleados en la determinación de la densidad, y destruyendo o corrigiendo las partes que no sean aceptables.

La experiencia ha demostrado que el inspector debe acompañar, siempre que se trate de estos ensayos, al cilindro compactador. Los cilindros han de poderse lastrar con cargas capaces de dar una sobrecarga por rueda de $11.300 \mathrm{~kg}$, siendo la presión interior de los neumáticos la que anteriormente se ha indicado. Como es natural, la carga de los cilindros y presión de los neumáticos depende del material y condiciones que se somete al ensayo. Para determinar la carga y presión óptima de neumáticos se han utilizado trozos experimentales.

\section{Evolución del espaldón}

En estos últimos veinte años se han venido utilizando muchos tipos de espaldones. Al principio de este período, la práctica más generalizada consistía en la construcción de un espaldón de materiales granulares, de $10 \mathrm{~cm}$ de espesor, que se extendía lateralmente a partir del borde del firme propiamente dicho de la calzada. A estos espaldones rudimentarios, ni se les consolidaba ni sellaba, y, por tanto, presentaban una serie de inconvenientes, tales como retención de agua, inestabilidad y dificultad de conservación.

Con posterioridad se puso en práctica el espaldón de tierra, especie de berma, repoblado con hierba hasta el borde del firme. Esta forma de operar se extendía tanto a las carreteras de primer orden como a las secundarias.

INFORMES DE LA CONSTRUCCION 101 


\section{espaldón normal de tierra}

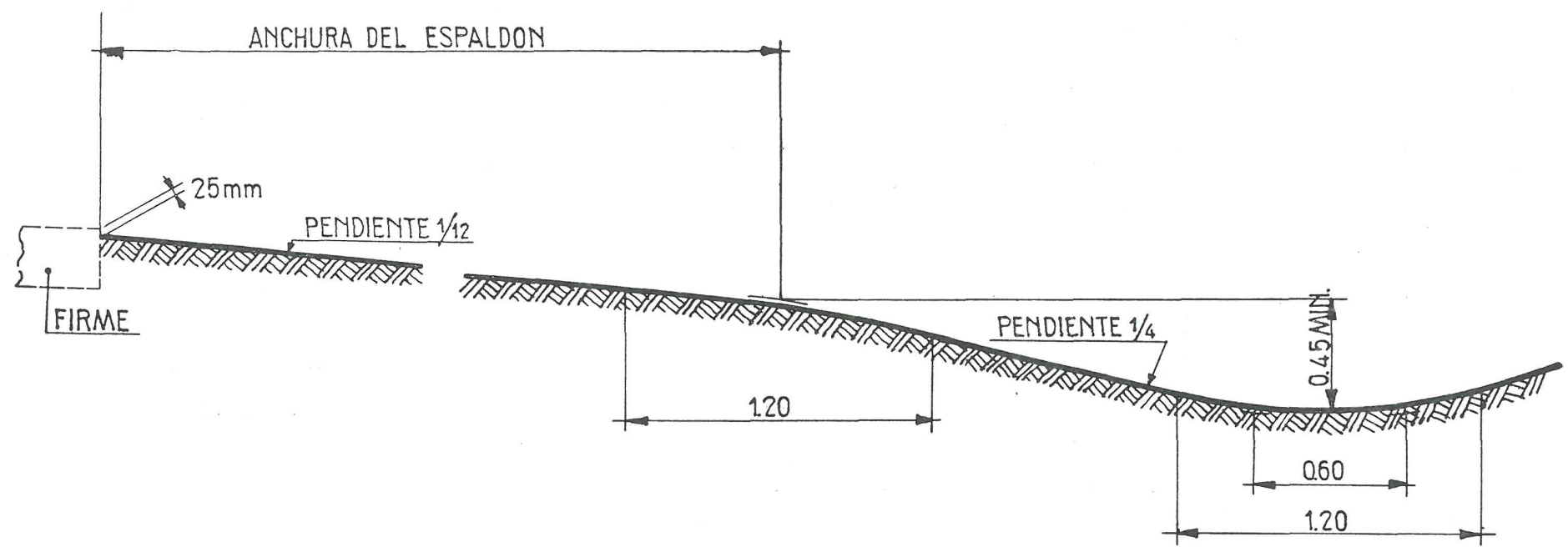

Aunque el aspecto estético de estos espaldones de césped era muy bonito, su estabilidad no era suficiente y la circulación sobre ellos era peligrosa en tiempos lluviosos.

La experiencia ha demostrado que el tráfico pesado llegaba a la rotura, incluso del mismo firme, por lo que, huyendo del espaldón, el conductor se paraba en el firme y entorpecía la circulación de otros vehículos.

En vista de todos estos inconvenientes, la Jefatura del Departamento de Ohio se lanzó a la construcción de espaldones debidamente estabilizados, que se extienden sólo a un lado en las carreteras subdivididas en dos direcciones únicas, y a los dos lados en las que la circulación es de más de 200 vehículos pesados por día. En las carreteras de menor circulación rodada, todavía se ven los espaldones de césped.

Las características más importantes de un espaldón para lograr un fácil y rápido drenaje del agua de lluvia, condición necesaria para la buena conservación de la calzada, consisten en terminar la superficie del espaldón en un plano $25 \mathrm{~mm}$ más bajo que el nivel del borde del firme, dar a su superficie una pendiente $1 / 12$ y prever una buena cuneta de pie, de unos $45 \mathrm{~cm}$ de profundidad y con suficiente sección para dar paso al agua que se pueda recoger.

\section{Espaldones estabilizados}

Actualmente, el tipo de espaldón normal utilizado por la Jefatura de Carreteras de Ohio, del que se acompaña un dibujo, tiene una anchura de $1,20 \mathrm{~m}$, un espesor de áridos machacados y compactados de $15 \mathrm{~cm}$ y una pendiente del $6 \%$. Los áridos pueden ser de escorias, piedra machacada o caliza. Estos materiales deben dejar, sin pasar por el tamiz americano núm. 4 , menos del $40 \%$. La granulometría exigida por esta Jefatura da como tamaño límite superior el de $50 \mathrm{~mm}$, dejando pasar de 0 a $15 \%$ en el tamiz núm. 200 y, además, tener un límite plástico no mayor de 6. Las características físicas de este material deben satisfacer, en lo que respecta a la compactación, las exigencias del ingeniero encargado de la construcción.

En uno de los dibujos que se acompañan, correspondiente al proyecto $36(1955)$, para la construcción de un firme flexible con espaldones estabilizados, de piedra machacada, se ha dado al espaldón un espesor de $15 \mathrm{~cm}$ y una pendiente $1 / 12$. Con objeto de mejorar el drenaje, en este caso, se ha prolongado la capa de cimiento del firme por debajo del espaldón, dándole una pendiente del $1,2 \%$. Bajo el espaldón estabilizado, el espesor de la capa de cimiento se ha recrecido hasta nivelarla con la parte inferior de la base del espaldón, con lo que se ha conseguido un espesor total de 38 a $45 \mathrm{~cm}$ de material granular en los $1,20 \mathrm{~m}$ de anchura del espaldón estabilizado.

En otro dibujo se ha representado el proyecto 184 (1953) para una carretera de dos direcciones únicas, independientes, de $14,5 \mathrm{~km}$ de longitud, cuya construcción se terminó en 1954. En esta carretera, los espaldones se extienden longitudinalmente a uno y otro lado de cada una de las dos direcciones únicas. En el fondo del espaldón se ha construído una zanja en cuyo fondo se ha colocado un tubo, rellenando el resto con materiales pétreos con gran proporción de huecos, con lo que se facilita el drenaje durante la construcción, y, posteriormente, en la explotación. Estos espaldones se han comportado muy bien, y apenas si requieren conservación.

También se han utilizado otros tipos de espaldones en esta Jefatura; entre ellos, alguno en los que se han empleado los cloruros de calcio y sodio con buenos resultados.

En un proyecto reciente para una carretera con una pendiente de 4 al $6 \%$ se han previsto espaldones estabilizados con cemento Portland. En otros proyectos de carreteras con pendientes similares, la erosión ha presentado problemas en los bordes; esto ha motivado 


\section{espaldón normal estabilizado}

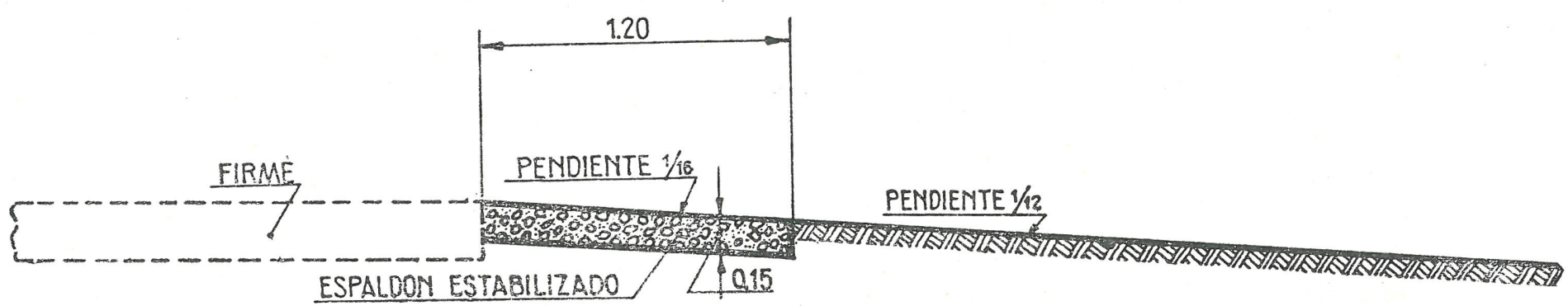

que, en este caso, se hayan proyectado espaldones estabilizados con cemento puesto que resisten mejor los efectos de la erosión. La cantidad de cemento que se ha previsto es del $10 \%$ en volumen. El resultado de esta práctica está todavía en observación, pero de momento es satisfactorio. Las dimensiones de estas estructuras son: de $1,20 \mathrm{~m}$ de anchura y $15 \mathrm{~cm}$ de espesor. La mezcla para el hormigonado de estos espaldones se puede realizar en la central o en la propia obra, compactándose y curándolo de una manera muy similar a los procedimientos empleados en la construcción de bases de firmes de suelo-cemento.

\section{Métodos constructivos}

Las excavaciones para la formación de la caja del espaldón se realizan siguiendo los métodos ordinarios, es decir, con ayuda de niveladoras, pero, para este caso, provistas de láminas apropiadas a las dimensiones de la caja que se ha de vaciar.

Una vez excavada la caja, se procede a la estabilización o compactación del fondo, ayudándose de un cilindro hasta lograr del 98 al $1.02 \%$ del procedimiento de la AASHO-T-99, de acuerdo con las condiciones del suelo que ha de consolidar. Cuanto más ligero es el peso relativo del suelo, tanto mayor es el grado de compactación exigido.

Para la compactación del suelo de apoyo se suele emplear el cilindro normal, pero también se puede realizar esta operación con otros tipos de cilindros, con tal que el resultado final sea el requerido. El control de la densidad del terreno compactado se obtiene por el procedimiento del ensayo de arena, es decir, conociendo el peso de los materiales sacados de un pocillo de prueba cuyo volumen se obtiene llenándolo con cierta cantidad de arena de densidad conocida, o, dicho de otra manera, utilizando algo así como una solución normal en ensayos químicos.

Preparada la caja y terminada su compactación, se procede a rellenarla con los áridos machacados, aplicándolos por tongadas que no excedan de $15 \mathrm{~cm}$ de espesor y compactándolas sucesivamente. Para esta clase de relleno se suele emplear una esparcidora mecánica de tipo ordinario. En una de las fotografías adjuntas se puede ver una de estas máquinas que, con objeto de dejar la carretera limpia, después de pasar la esparcidora, ésta va provista de una escoba mecánica acoplada en la parte posterior de la esparcidora.

La Jefatura de Ohio permite cualquier clase de material auxiliar para la compactación inicial, pero para

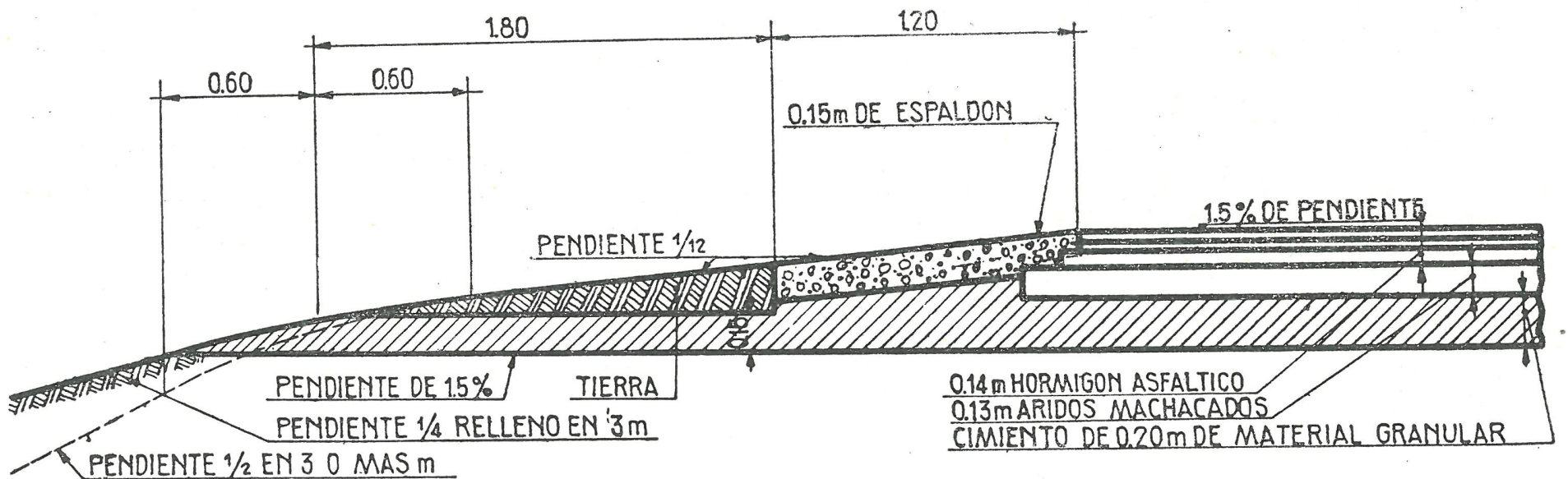

\section{espaldón perfeccionado para el proyecto 36}




\section{espaldón con dren para el proyecto 184}

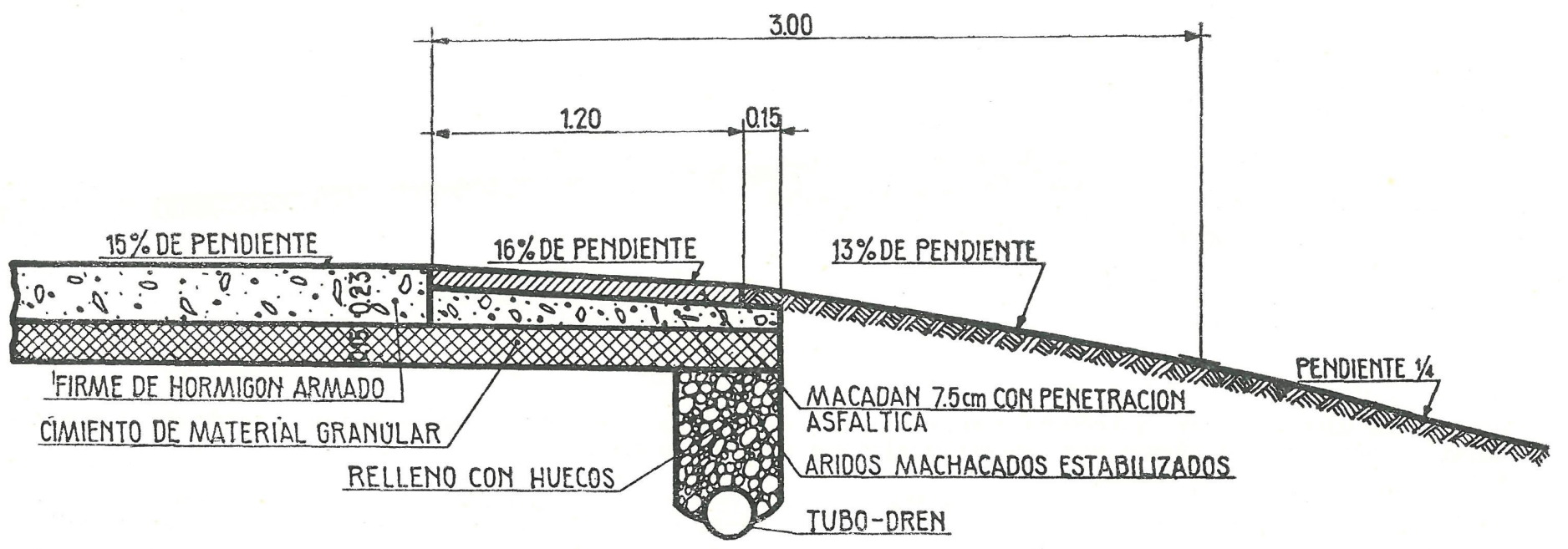

la consolidación en su fase final se requiere el uso del cllindro de ruedas neumáticas de características fijadas previamente. En una de las figuras se ha representado el tipo que exige la Jefatura para la compactación final.

Para controlar esta operación se siguen los procedimientos basados en el principio de densidad-humedad. Algunas veces, y a juicio del ingeniero encargado, se permite el riego con agua para facilitar esta operación y evitar la segregación o separación. La densidad exigida se basa en ensayos previos de esta clase sobre el terreno. Como se dijo anteriormente, la compactación se controla con los ensayos de densidad siguiendo el método de arena, no dándose por satisfecho hasta que la estabilización ha alcanzado el grado de densidad, o mayor, al correspondiente a la humedad óptima en el método de AASHO-T-99, y utilizando materiales que han pasado por un tamiz de malla de $19 \mathrm{~mm}$ de lado.

El número de ensayos necesarios para el control de compactación durante la construcción, si la ejecución se desarrolla lentamente y con materiales uniformes, no es muy grande.

Si se van obteniendo densidades adecuadas y la humedad se mantiene satisfactoriamente, la función de la inspección se limita a fijar el número de pasadas que ha de dar el cilindro, así como comprobar que estas pasadas se han realizado. Bajo estas condiciones, basta con uno o dos ensayos de densidad diariamente.

\section{Problemas constructivos}

Fia de lograrse un control eficaz de la compactación del terreno y espaldón si queremos obtener una estabilización satisfactoria.

La forma de los espaldones y cunetas ha de ser la más apropiada durante el período de construcción, con objeto de lograr un buen drenaje, aun con lluvias fuertes, y evitar que se formen charcas en el firme. Durante la ejecución han de preverse cunetas de drenaje a través de los espaldones, con objeto de lograr un drenaje eficaz. Parece que existe una tendencia general a no abrir estas cunetas a través de los espaldones, por lo que el ingeniero encargado llevará su atención a esta previsión en los lugares donde se deban establecer y mantener.

Para mejorar las prácticas hasta hoy extendidas en la ejecución de firmes flexibles, se ha propuesto mantener todo el material auxiliar pesado $\mathrm{y}$ grandes camiones utilizados para el transporte fuera de la subrasante compactada, cimiento y capas de la base durante el período de ejecución, así como exigir a la maquinaria del equipo no necesitada en la construcción del firme que no pase sobre los espaldones. Se puede anticipar que estas exigencias crean problemas en la construcción de los espaldones. La circulación del material pesado sobre los espaldones durante la construcción del pavimento constituye una amenaza grave para el mantenimiento de las cunetas transversales a través de los espaldones y la formación de rodadas, que hace difícil la conservación en la debida forma para lograr un drenaje adecuado del agua de superficie durante la construcción.

En las carreteras del departamento de Ohio, sometidas a una gran circulación pesada, existe una necesidad incontestable de espaldones estables, pavimentados convenientemente, para efectuar un drenaje del agua de superficie.

Actualmente, y de acuerdo con las posibilidades, la Jefatura de Carreteras de Ohio está desarrollando un programa para el tratamiento superficial bituminoso de aquellos espaldones que se han conservado muchos años en un estado original de compactación.

De acuerdo con las necesidades de las carreteras actuales de primer orden, en los proyectos de nueva planta se debe prever el tratamiento superficial bituminoso de los espaldones estabilizados.

J. J. U. 